\title{
Prognostic Values of the Gray-to-White Matter Ratio on Brain Computed Tomography Images for Neurological Outcomes after Cardiac Arrest: A Meta-Analysis
}

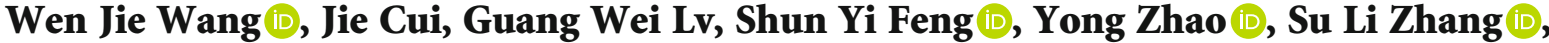 \\ and Yong $\mathrm{Li}$
}

Emergency Department, Cangzhou Central Hospital, No. 16 Xinhua Road, Yunhe Qu, Cangzhou 061000, China

Correspondence should be addressed to Yong Li; ly13333367871@hotmail.com

Received 8 July 2020; Revised 7 September 2020; Accepted 22 October 2020; Published 3 November 2020

Academic Editor: Alessandro Martorana

Copyright (C) 2020 Wen Jie Wang et al. This is an open access article distributed under the Creative Commons Attribution License, which permits unrestricted use, distribution, and reproduction in any medium, provided the original work is properly cited.

\begin{abstract}
Background and Purpose. The gray-to-white matter ratio (GWR) on brain computed tomography (CT) is associated with neurological outcomes after cardiac arrest (CA); however, the prognostic value of GWR in CA patients has yet to be confirmed. Therefore, we conducted a meta-analysis of related studies to investigate the prognostic value of GWR on brain CT for neurological outcomes after CA. Materials and Methods. The PubMed, ScienceDirect, Web of Science, and China National Knowledge Infrastructure databases were searched for all relevant articles published before March 31, 2020, without any language restrictions. The pooled odds ratios (ORs) and $95 \%$ confidence intervals (CIs) were calculated with a random-effects model using Stata 14.0 software. Result. A total of 24 eligible studies with 2812 CA patients were recruited in the meta-analysis. The pooled result showed that decreased GWR was correlated with poor neurological outcomes after CA $(\mathrm{OR}=11.28,95 \% \mathrm{CI}$ : 6.29-20.21, and $P<0.001)$ with moderate heterogeneity $\left(I^{2}=71.5 \%, P<0.001\right)$. The pooled sensitivity and specificity were 0.58 (95\% CI: $0.47-0.68)$ and 0.95 (95\% CI: 0.87-0.98), respectively. The area under the curve (AUC) of GWR was 0.84 (95\% CI: 0.80-0.87). Compared with GWR (cerebrum) and GWR (average), GWR using the basal ganglion level of brain CT had the highest AUC of $0.87(0.84-0.90)$. Subgroup analysis indicated that heterogeneity may be derived from the time of CT measurement, preset specificity, targeted temperature management, or proportion of cardiac etiology. Sensitivity analysis indicated that the result was stable, and Deeks' plot showed no possible publication bias $(P=0.64)$. Conclusion. Current research suggests that GWR, especially using the basal ganglion level of brain CT, is a useful parameter for determining neurological outcomes after CA.
\end{abstract}

\section{Introduction}

Cardiac arrest (CA) is a growing public health issue carrying an enormous global burden of morbidity, and out-ofhospital CA accounts for approximately $10 \%$ of individuals who survive to hospital discharge [1]. Unfortunately, the incidence of neurological sequelae among survivors of CA remains as high as $70 \%$ despite advances in post-CA care $[2,3]$. Neurological sequelae have a significantly negative impact on the community in terms of life years lost and healthcare costs in survivors and the emotional burden of family members. Early clinical predictors in survi- vors of $\mathrm{CA}$ are important for counseling families and making management decisions.

Some signs on brain computed tomography (CT) that have been associated with ischemic cerebral insult include a loss of boundary between the gray matter (GM) and the white matter (WM). Several studies [4-27] indicate that a loss of differentiation between GM and WM, reflected as decreased gray-to-white matter ratio (GWR) on brain CT, predicts a poor outcome in CA patients. However, in the absence of large and comprehensive diagnostic studies, the prognostic role of GWR in survivors of CA is still controversial. Therefore, we performed a meta-analysis to evaluate 


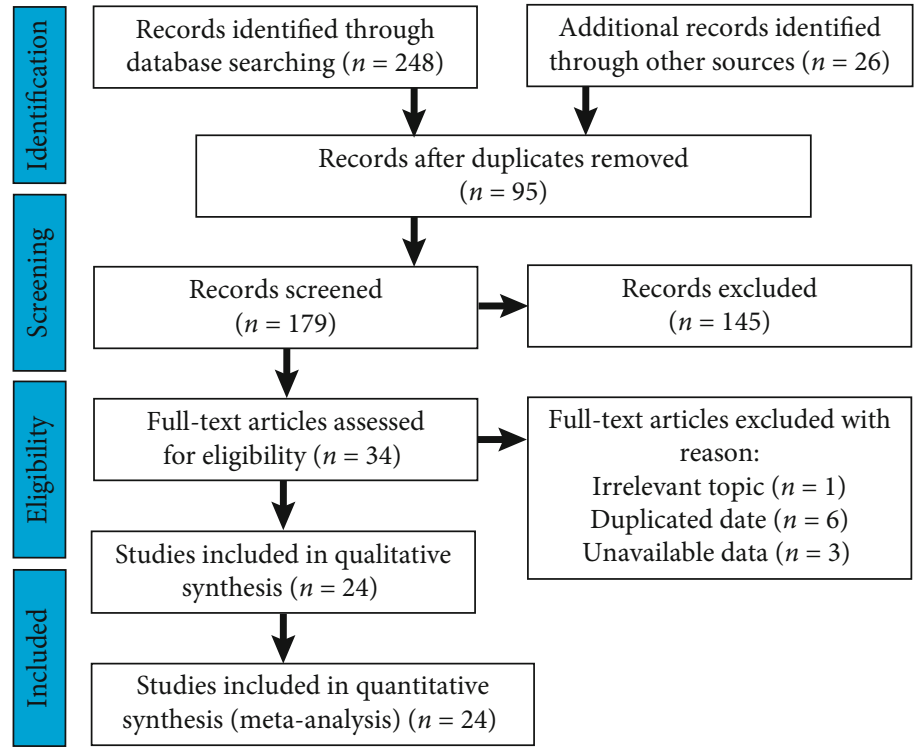

FIGURE 1: Flow diagram showed the selection process of meta-analysis.

the prognostic value of GWR for neurological outcomes after CA.

\section{Methods}

This meta-analysis was carried out according to the Preferred Reporting Items for Systematic Reviews and Meta-Analyses statement [28]. We registered our systematic review prospectively in PROSPERO (CRD42020182066). As this study was a review of published literature, the approval of an ethics committee and consent of patients were not required.

2.1. Literature Search. The PubMed, ScienceDirect, Web of Science, and China National Knowledge Infrastructure databases were searched for all relevant articles published before March 31, 2020, without any language restrictions. The search terms included "gray-to-white ratio" and "cardiac arrest." Moreover, the references from the retrieved studies were also reviewed by manual search to identify any new eligible studies.

Studies were included if they satisfied the following criteria: the full-text publication evaluated the association of GWR on brain CT for neurological outcomes, and sufficient information should be available to evaluate odds ratios (ORs) with 95\% confidence intervals (CIs). Letters, comments, editorials, case reports, communications, and duplicated studies were excluded.

2.2. Data Extraction and Quality Assessment. The data extraction of the present study was independently performed by two reviewers. The extracted data included the first names of the author, country, study design, sample size, time of brain $\mathrm{CT}$ measurement, targeted temperature management (TTM), area under the curve (AUC), cutoff value, GWR value, proportion of cardiac etiology, and outcomes for each study. The caudate nucleus $(\mathrm{CN})$, putamen (PU), posterior limb of the internal capsule (PLIC), and corpus callosum (CC) were measured for the basal ganglia, and the gray and white matter from the medial cortex was measured at the centrum semiovale (MC1, MW1) and high cortical level (MC2, MW2). The GWR for the basal ganglia $(\mathrm{GWR}-\mathrm{BG}=(\mathrm{CN}+\mathrm{PU}) /(\mathrm{PLIC}+\mathrm{CC})), \mathrm{GWR}$ for the cerebrum $(\mathrm{GWR}-\mathrm{CE}=(\mathrm{MC1}+\mathrm{MC} 2) /(\mathrm{MW} 1+\mathrm{MW} 2))$, and the average of the two (GWR-AV $=(\mathrm{GWR}-\mathrm{BG}+\mathrm{GWR}-\mathrm{CE}) / 2)$ were calculated as previously described [4]. The simplified GWR estimation method (GWR-SI = PU/PLIC, CN/PLIC, or PU/CC) was calculated as previously described [7].

Quality Assessment of Diagnostic Accuracy Studies 2 was used to assess the reporting quality of the included original studies [29]. If there was disagreement, a consensus was reached by a third reviewer.

2.3. Statistical Analysis. Statistical analyses will be performed using Stata/MP 14.0 (StataCorp, College Station, TX, USA). The associations between GWR and neurological outcomes after CA were estimated on the basis of the pooled ORs and $95 \%$ CIs. Heterogeneity was assessed by using the $I^{2}$ statistic where $P<0.1$ and/or $I^{2}>50 \%$ indicated heterogeneity between the data, and the combined analysis was performed using a random-effects model. Otherwise, a fixed-effects model was used. Publication bias was formally assessed using Deeks' plot if more than 10 qualified studies are included in our study. Additionally, we used sensitivity analysis to evaluate the stabilization of the study. For all statistical analyses, $P<0.05$ was considered to indicate statistical significance, and all tests were two-sided.

\section{Results}

3.1. Literature Search. The general characteristics of the included studies are summarized in Figure 1. Initially, 274 publications were retrieved by the mentioned search strategy, of which 95 duplicated studies were excluded. Of the remaining 179 studies, 155 were excluded for being 


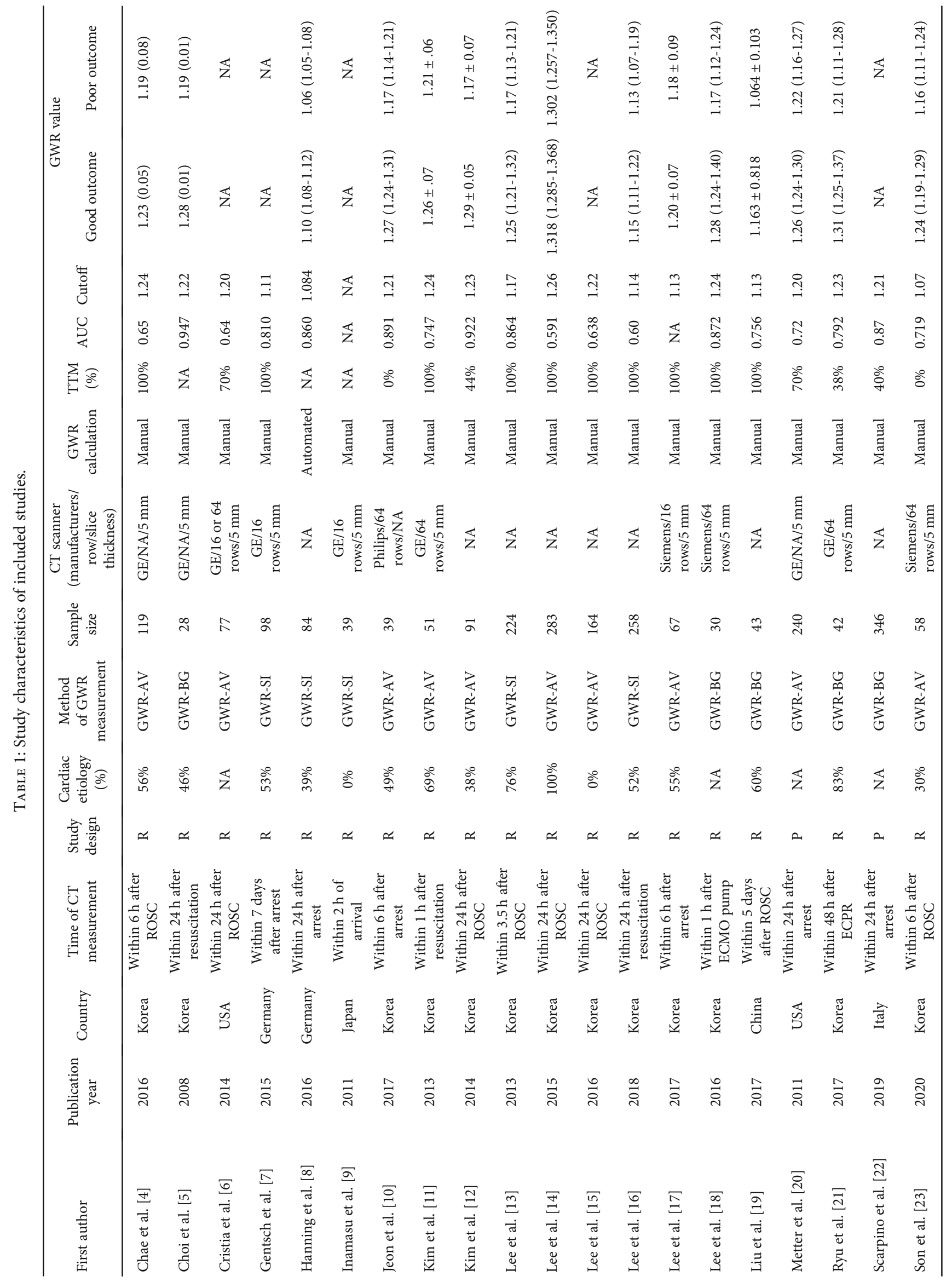




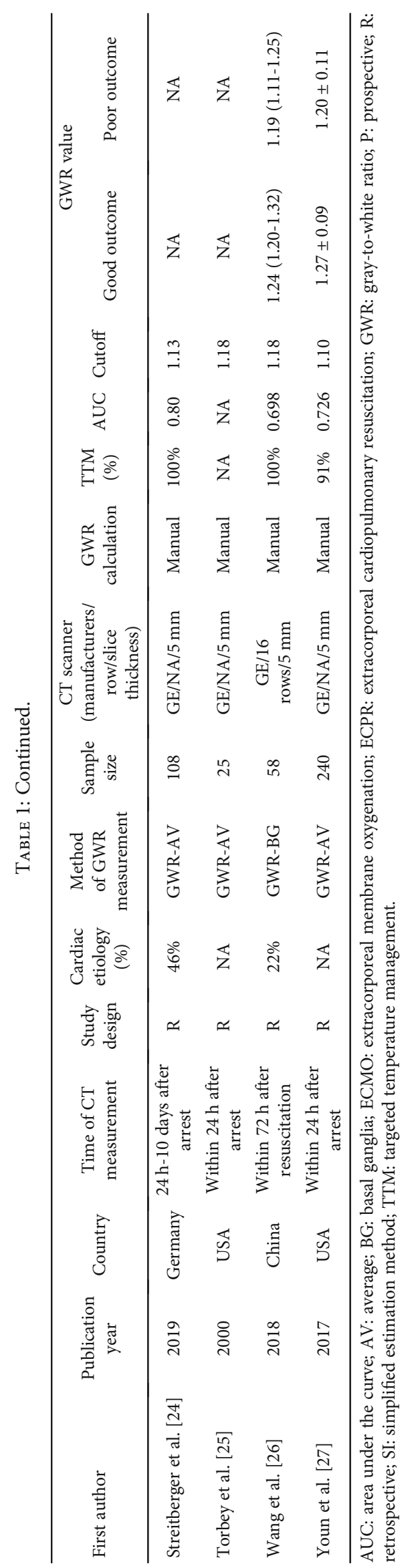




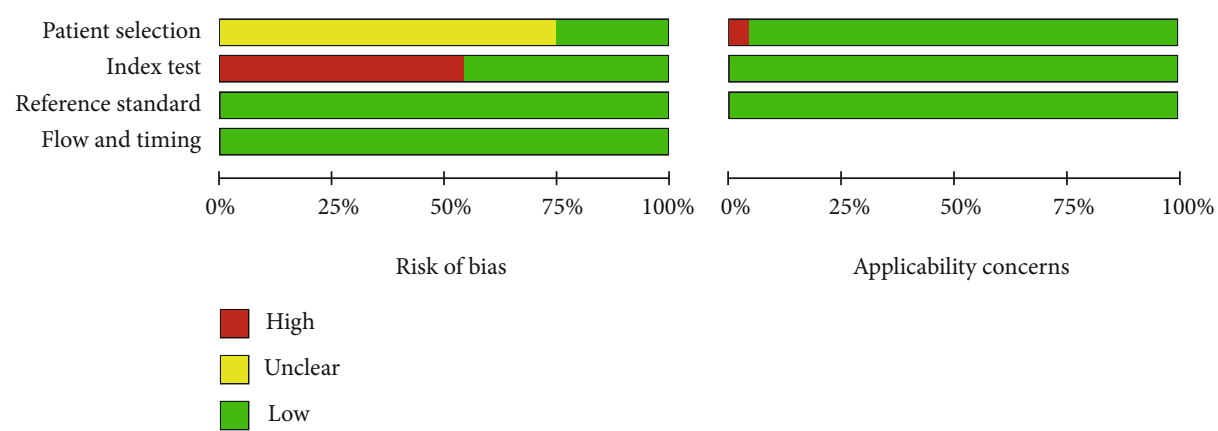

FIGURE 2: Flow diagram of studies' selection and quality assessment of the included articles.

\begin{tabular}{|c|c|c|c|}
\hline \multicolumn{2}{|l|}{$\begin{array}{l}\text { Study } \\
\text { ID }\end{array}$} & \multirow{2}{*}{$\begin{array}{c}\text { OR }(95 \% \mathrm{CI}) \\
3.15(1.45,6.85)\end{array}$} & \multirow{2}{*}{$\begin{array}{c}\begin{array}{c}\% \\
\text { Weight }\end{array} \\
6.56\end{array}$} \\
\hline Chae 2016 & - & & \\
\hline Choi 2008 & & $31.67(1.60,626.21)$ & 2.52 \\
\hline Cristia 2014 & & $4.45(1.51,13.11)$ & 5.92 \\
\hline Gentsch 2015 & & $59.78(3.51,1017.97)$ & 2.70 \\
\hline Hanning 2016 & & $33.47(9.09,123.22)$ & 5.41 \\
\hline Inamasu 2011 & & $99.00(4.47,2194.36)$ & 2.40 \\
\hline Jeon 2017 & & $43.24(2.22,840.32)$ & 2.54 \\
\hline Kim 2013 & 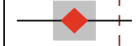 & $4.38(1.31,14.68)$ & 5.63 \\
\hline Kim 2014 & & $235.00(13.30,4152.16)$ & 2.65 \\
\hline Lee 2013 & & $184.56(11.23,3033.47)$ & 2.74 \\
\hline Lee 2015 & - & $2.91(1.58,5.37)$ & 6.86 \\
\hline Lee 2016 & & $15.49(0.91,262.47)$ & 2.70 \\
\hline Lee 2018 & - & $2.08(1.22,3.54)$ & 6.99 \\
\hline Lee 2017 & H & $2.40(0.61,9.53)$ & 5.25 \\
\hline Lee 2016 & & $70.71(3.17,1579.77)$ & 2.39 \\
\hline Liu 2017 & & $6.56(1.59,27.07)$ & 5.16 \\
\hline Metter 2011 & 1 & $23.85(5.65,100.72)$ & 5.11 \\
\hline Ryu 2017 & & $8.30(1.87,36.83)$ & 5.00 \\
\hline Scarpino 2019 & & $236.21(14.51,3844.81)$ & 2.75 \\
\hline Son 2020 & & $12.05(0.65,224.96)$ & 2.59 \\
\hline Streitberger 2019 & & $17.83(3.98,79.88)$ & 4.98 \\
\hline Torbey 2000 & & $21.67(1.06,442.00)$ & 2.49 \\
\hline Wang 2018 & 1 & $7.00(1.41,34.68)$ & 4.76 \\
\hline Youn 2017 & -1 & $4.15(0.54,31.87)$ & 3.90 \\
\hline Overall $(I$-squared $=71.5 \%, \mathrm{p}<0.001)$ & & $11.28(6.29,20.21)$ & 100.00 \\
\hline Note: weights are from random effects analysis & & & \\
\hline $\begin{array}{c} \\
0.00024\end{array}$ & 1 & & \\
\hline
\end{tabular}

FiguRE 3: Forest plots for the meta-analysis of the prognostic value of the gray-to-white matter ratio for neurological outcomes after cardiac arrest.

reviews, comments, or abstracts and unrelated to the topic. After screening the title, abstract, and full text, 24 studies [4-27] that involved a total of 2812 patients were eligible for inclusion.

3.2. Characteristics of the Included Studies. The characteristics of the included studies are summarized in Table 1. Of the 24 studies [4-27], 22 were retrospective cohort studies [4-19, 21, 23-27], and two were prospective in nature $[20,22]$. The number of included patients per study ranged from 25 to 346 , with an average of 115 . The reported cutoff of GWR in the included studies varied between 1.07 and 1.26. The AUC of GWR in different studies varied between 0.650 and 0.947 .

3.3. Quality Assessment. The patient selection risk of bias domain in 18 studies $[4,5,7,9-11,13-21,23,24,26]$ was labeled as unclear risk because the authors did not indicate whether patients were recruited consecutively, and the index text risk of bias domain in 13 studies $[4,6,8,11,14,16,17$, 19-21, 24, 26, 27] was labeled high risk because the authors did not have a preset specificity. In addition, the patient 


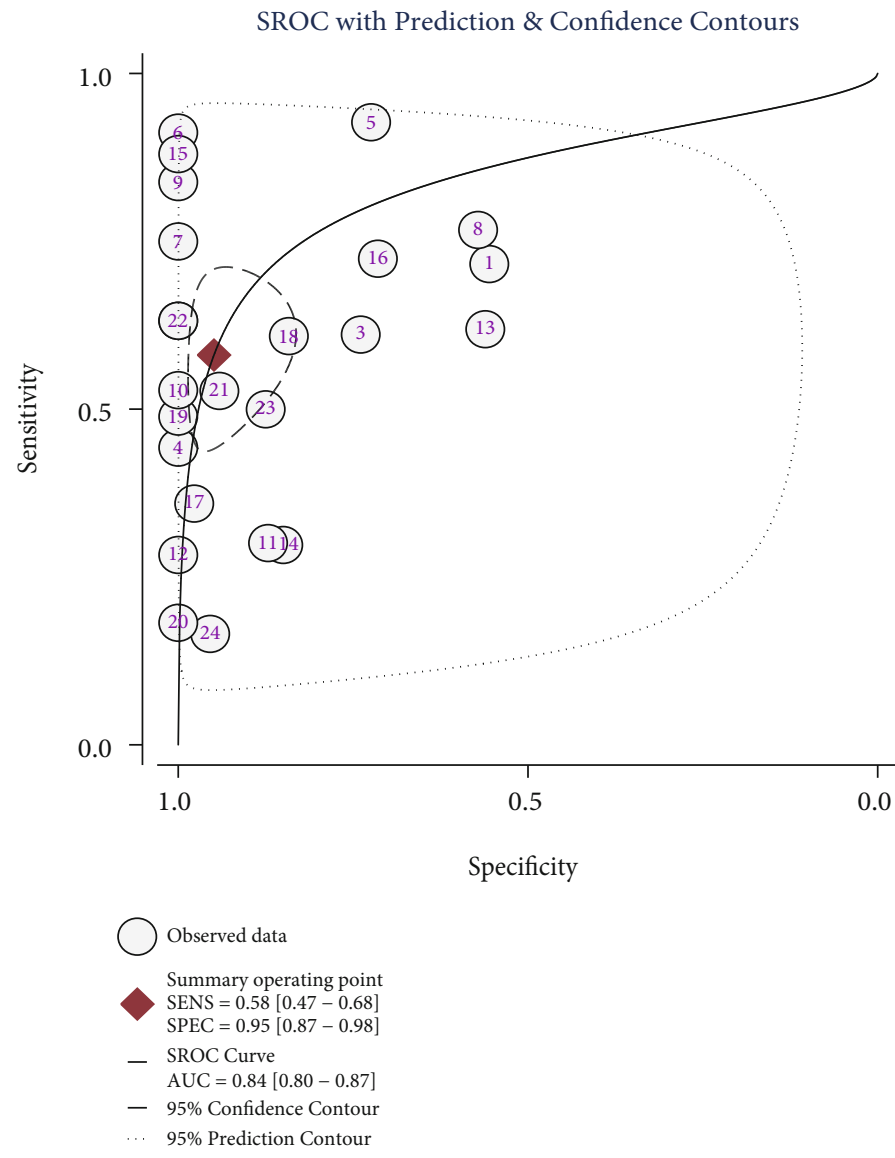

FIGURE 4: Summary ROC curve for estimating the testing accuracy of the gray-to-white matter ratio for neurological outcomes after cardiac arrest.

TABle 2: Prognostic performance of GWR using different regions of the brain.

\begin{tabular}{lcccccc}
\hline & OR $(95 \% \mathrm{CI})$ & $P$ & $I^{2}(P$ value $)$ & Sensibility & Specificity & AUC \\
\hline GWR-BG & $15.77(6.57-37.71)$ & $<0.001$ & $0 \%(0.690)$ & $0.32(0.12-0.60)$ & $1.0(0.13-1.0)$ & $0.87(0.84-0.90)$ \\
GWR-CE & $11.06(4.68-26.16)$ & $<0.001$ & $0 \%(0.947)$ & $0.27(0.14-0.45)$ & $1.0(0.24-1.00)$ & $0.72(0.68-0.76)$ \\
GWR-AV & $15.92(6.88-36.83)$ & $<0.001$ & $59.6 \%(0.722)$ & $0.35(0.18-0.58)$ & $1.0(0.14-1.00)$ & $0.79(0.75-0.82)$ \\
\hline
\end{tabular}

AUC: area under the curve; AV: average; BG: basal ganglia; CE: cerebrum; CI: confidence interval; GWR: gray-to-white ratio; OR: odds ratio.

selection risk of bias domain in one study [25] was labeled as unclear risk because patients with a normal brain CT scan served as controls (Figure 2 and additional Figure 1).

3.4. Diagnostic Performance. As shown in Figure 3, the pooled results demonstrated that decreased GWR was associated with poor prognosis in post-CA patients $(\mathrm{OR}=11.28$, 95\% CI: 6.29-20.21, and $\left.P<0.001 ; I^{2}=71.5 \%, P<0.001\right)$. As shown in Figure 4, the pooled sensitivity and specificity were 0.58 (95\% CI: $0.47-0.68$ ) and 0.95 (95\% CI: $0.87-$ $0.98)$, respectively. The positive likelihood ratio was 11.4 (95\% CI: 4.60-28.40), the negative likelihood ratio was 0.44 (95\% CI: 0.35-0.56), the diagnostic OR was 26 (95\% CI: 10-69), and the AUC was 0.84 (95\% CI: 0.80-0.87).

Eight studies [7, 11, 14, 15, 18, 19, 21, 26] compared the prognostic performance of GWR-BG, GWR-CE, and GWR-AV. The pooled results (Table 2) demonstrated that
GWR using the basal ganglion level of brain CT has the highest AUC of $0.87(0.84-0.90)$.

3.5. Subgroup Analysis. Due to heterogeneity, subgroup analysis was conducted on the basis of the preset specificity (preset specificity $=1$ or nonpreset specificity), sample size $(<100$ or $\geq 100$ ), study design (retrospective or prospective), country (Asia or non-Asia), time of CT measurement ( $\leq 24 \mathrm{~h}$ or $>24 \mathrm{~h}$ ), TTM (all or not all), cutoff ( $\leq 1.18$ or $>1.18$ ), cardiac etiology ( $\leq 50 \%$ or $>50 \%$ ), and row of CT (16 rows or 64 rows) for a subsequent investigation of potential heterogeneity. We found that nonpreset specificity, time of CT measurement, TTM, or proportion of cardiac etiology may cause heterogeneity but did not affect the final conclusion (Table 3 ).

3.6. Sensitivity Analysis and Publication Bias Assessment. Sensitivity analysis did not find any single study that had 
TABLE 3: Subgroup analysis.

\begin{tabular}{|c|c|c|c|c|}
\hline & $N$ & OR $(95 \% \mathrm{CI})$ & $P$ & $I^{2}(P$ value $)$ \\
\hline \multicolumn{5}{|l|}{ Specificity } \\
\hline 1 & 11 & $58.90(24.40-142.22)$ & $<0.001$ & $0.0 \%(0.852)$ \\
\hline Not 1 & 13 & $5.44(3.33-8.88)$ & $<0.001$ & $60.5 \%(0.002)$ \\
\hline \multicolumn{5}{|l|}{ Sample size } \\
\hline$<100$ & 15 & $12.08(6.32-23.06)$ & $<0.001$ & $42.6 \%(0.041)$ \\
\hline$\geq 100$ & 9 & $9.37(3.55-24.74)$ & $<0.001$ & $83.4 \%(<0.001)$ \\
\hline \multicolumn{5}{|l|}{ Study design } \\
\hline Retrospective & 22 & $9.23(5.31-16.06)$ & $<0.001$ & $65.8 \%(<0.001)$ \\
\hline Prospective & 2 & $57.46(5.18-637.15)$ & 0.001 & $59.7 \%(0.115)$ \\
\hline \multicolumn{5}{|l|}{ Country } \\
\hline Asia & 16 & $7.81(4.15-14.68)$ & $<0.001$ & $65.7 \%(<0.001)$ \\
\hline Non-Asia & 8 & $18.26(7.30-45.68)$ & $<0.001$ & $53.2 \%(0.037)$ \\
\hline \multicolumn{5}{|l|}{ Cutoff } \\
\hline$\leq 1.18$ & 11 & $10.16(4.00-25.76)$ & $<0.001$ & $73.6 \%(<0.001)$ \\
\hline$>1.18$ & 12 & $11.72(5.12-26.83)$ & $<0.001$ & $72.0 \%(<0.001)$ \\
\hline \multicolumn{5}{|c|}{ Time of CT measurement } \\
\hline$\leq 24 \mathrm{~h}$ & 19 & $11.75(5.84-23.66)$ & $<0.001$ & $75.8 \%(<0.001)$ \\
\hline$>24 \mathrm{~h}$ & 5 & $10.25(4.97-21.14)$ & $<0.001$ & $0 \%(0.555)$ \\
\hline \multicolumn{5}{|l|}{ TTM } \\
\hline All & 12 & $5.96(3.18-11.16)$ & $<0.001$ & $66.0 \%(0.001)$ \\
\hline Part & 6 & $17.79(4.56-69.39)$ & $<0.001$ & $72.0 \%(0.003)$ \\
\hline None & 2 & $22.63(2.82-181.78)$ & $<0.001$ & $0.0 \%(0.547)$ \\
\hline \multicolumn{5}{|l|}{ Cardiac etiology } \\
\hline$\leq 50 \%$ & 8 & $21.97(10.86-44.46)$ & $<0.001$ & $0.0 \%(0.593)$ \\
\hline$>50 \%$ & 9 & $4.51(2.44-8.34)$ & $<0.001$ & $64.4 \%(0.004)$ \\
\hline \multicolumn{5}{|l|}{ Row of CT } \\
\hline 16 rows & 4 & $11.66(2.22-11.66)$ & 0.004 & $61.6 \%(0.050)$ \\
\hline 64 rows & 5 & $8.76(3.65-21.01)$ & $<0.001$ & $6.1 \%(0.372)$ \\
\hline
\end{tabular}

CI: confidence interval; CT: computed tomography; OR: odds ratio; TTM: targeted temperature management.

an impact on the total pooled effect, indicating that the result was stable (Figure 5). Publication bias was examined using Deeks' plot asymmetry test, and the funnel plot did not reveal significant publication bias $(P=0.64$; Figure 6$)$.

\section{Discussion}

In this systematic review and meta-analysis, we evaluated the diagnostic accuracy of GWR for neurological outcomes after CA by analyzing the current clinical evidence. Our principal findings were as follows: the predictive ability of GWR for a poor neurological outcome assessed using the AUC was 0.84 (95\% CI: 0.80-0.87). Furthermore, GWR using the basal ganglion level of brain CT had the highest AUC of 0.87 (0.84-0.90).

A previous meta-analysis by Lopez Soto et al. [30] showed that a decreased GWR brain CT is useful for predicting poor neurological outcomes with a sensitivity of 0.44 and specificity of 0.97 ; this finding is consistent with our results. However, we comprehensively searched the PubMed, ScienceDirect, Web of Science, and China National Knowledge Infrastructure databases, and four additional studies were considered in the present meta-analysis. In addition, we compared the prognostic performance of GWR measurement methods, including GWR-BG, GWR-CE, and GWR$\mathrm{AV}$, and discovered that the GWR using GWR-BG had the highest performance.

The AUC of GWR-BG was higher than those of GWR$\mathrm{CE}$ and GWR-AV for the following reasons. The relative attenuations of GM and WM throughout various regions of the brain show discrepancy. Gentsch et al. [7] speculated that the basal ganglia are more severely damaged by hypoxia due to their high metabolic activity and their location within the boundary zones of perfusion or measurement of Hounsfield units in the cortical GM is less reliable due to partial volume effects [31].

There is currently no consensus on a distinct GWR cutoff value that may predict a poor outcome with high specificity. The reported cutoff of GWR in the 24 included studies varied between 1.07 and 1.26. In addition, the AUC of GWR showed great discrepancy in different studies and varied between 0.650 and 0.947 . This discrepancy may be associated with the following reasons. First, Morimoto et al. [32] reported that cerebral edema is more common after CA of respiratory 


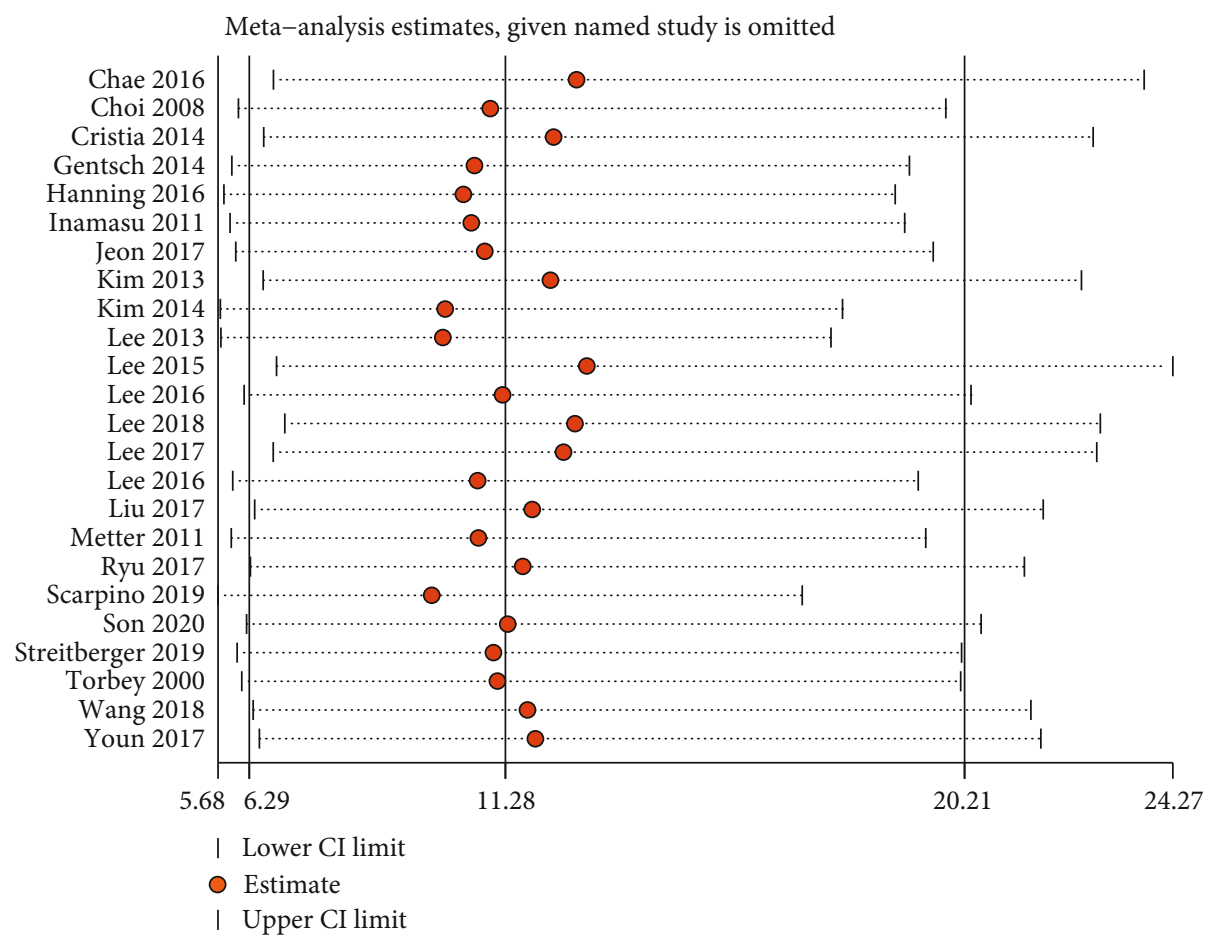

FIgURE 5: Sensitivity analysis to examine the influence of each individual study on the overall results.

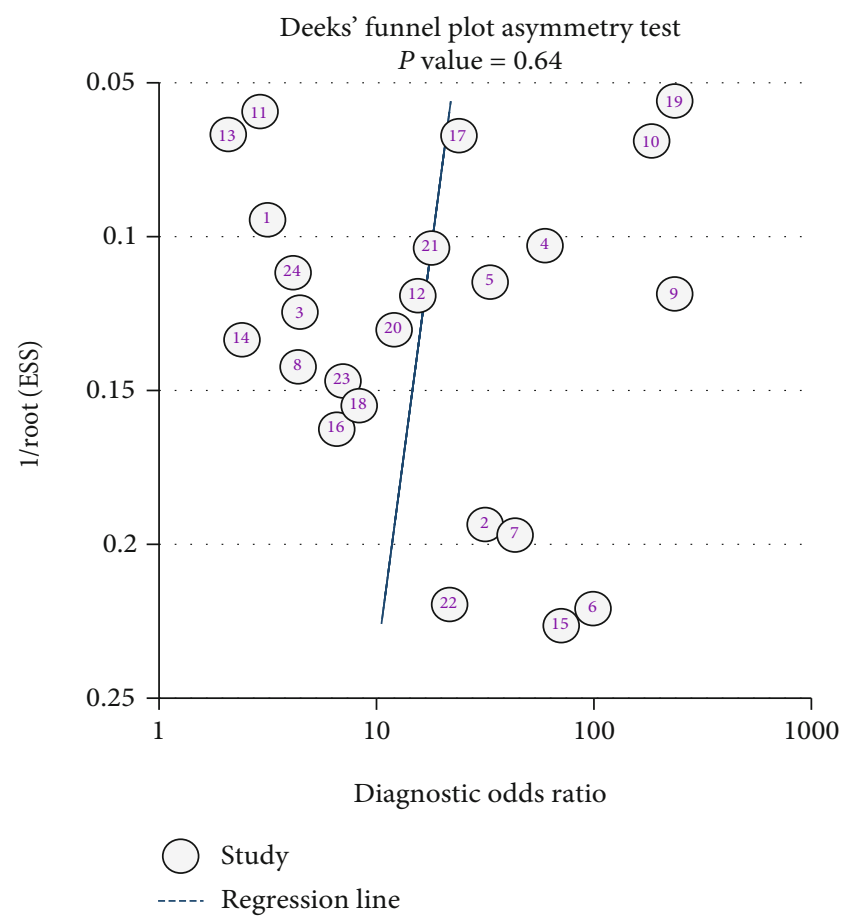

FIGURE 6: Deeks' plot for the publication bias test.

etiology due to the development of metabolic acidosis (possibly lactic acidosis) induced by hypoxia. Similarly, Lee et al. [15] suggested that noncardiac etiology is associated with a more severe brain edema than cardiac etiology. A change in GWR values is associated with the water content of the brain tissue; therefore, patients with respiratory or noncardiac eti- ology CA may have lower GWR in the initial brain CT. Second, the timing for brain CT scans was not standardized.

Subgroup analysis indicated that nonpreset specificity, time of CT measurement, TTM, and proportion of cardiac etiology may cause heterogeneity. Although conclusions based on pooled estimates in subgroups are consistent, clinicians should consider these heterogeneity factors in their clinical practice. Several studies $[5,7,9,10,12,13,15,18$, $22,23,25]$ have determined cutoff values with $100 \%$ specificity for predicting poor neurological outcomes to identify patients with a minimal chance of achieving a good neurological outcome, which could partly affect the difficulty of deriving conclusions based on pooled estimates. Survival with a favorable neurological outcome may be possible in patients with hypothermia despite a severe early injury, which influences the predictive value GWR for the neurological outcome. Thus, the reevaluation of the predictive value of GWR is necessary when patients receive hypothermia therapy. Metter et al. [20] proved that the time from arrest to CT is not related to GWR or attenuation values although cerebral edema after CA evolves over time. Notably, brain CT scans performed during the first $2 \mathrm{~h}$ after ROSC may not provide sufficient time for the formation of cerebral edema and GWR within this time window and are not a good outcome predictor $[15,33]$. Noncardiac etiology, instead of cardiac etiology, may lead to severe brain injury with an eventual poor clinical outcome; GWR is more helpful in predicting neurological outcomes in CA patients with noncardiac etiologies than with cardiac etiologies [15]. However, we failed to perform a subgroup analysis on the basis of the slice thickness of brain CT scanners because only one study used $2.5-4.8 \mathrm{~mm}$ slice thickness; the others used $5 \mathrm{~mm}$ slice 
thickness. However, Oh et al. [34] obtained a significant variance in Hounsfield units when different CT scanners were used but observed only minor differences in GWR values. Hanning et al. [8] utilized coregistration with an atlas to calculate the average GWR, but the obtained value differs from the average GWR calculated through the manual placement of a few regions of interest. This condition indicates that the computing method, whether automated or manual, needs to be considered when assessing the predictive value of GWR.

Several limitations should be carefully considered in the present study. First, our analysis was based mainly on findings from retrospective studies, which might contain a higher number of confounding factors than prospective studies. Second, the studies involved in this meta-analysis had varying cutoff values. Third, the included studies exhibited significant heterogeneity, which may have reduced the reliability of the analysis. Fourth, neuroprognostication studies are potentially susceptible to a self-fulfilling prophecy because the investigated prognostic parameters may affect the withdrawal of life-sustaining therapy. Lastly, the timing of brain CT is critical to the sensitivity of GWR to poor outcome prediction, and some included studies did not perform CT at optimal time points (within $6 \mathrm{~h}$ and usually around $2 \mathrm{~h}$ ) [17]. Further research is needed to establish the optimal timing of brain CT measurement.

\section{Conclusions}

Brain CT is simple, cost-effective, and easily implemented after CA. Stratification analysis based on CT scan obtained at different time points after CA, etiology induced by CA, and therapeutic hypothermia is required to understand the pattern of GWR and neurological outcomes.

\section{Abbreviations}

AUC: Area under the curve

CA: Cardiac arrest

CI: Confidence interval

GM: Gray matter

GWR: Gray-to-white ratio

OR: Odds ratio

TTM: Targeted temperature management

WM: White matter.

\section{Data Availability}

The datasets used and/or analyzed during the current study are available from the corresponding author on reasonable request.

\section{Conflicts of Interest}

The authors declare that they have no competing interests.

\section{Supplementary Materials}

Supplementary Fig 1: methodological quality summary. (Supplementary materials)

\section{References}

[1] D. Mozaffarian, E. J. Benjamin, A. S. Go et al., "Executive Summary: Heart Disease and Stroke Statistics-2016 Update," Circulation, vol. 133, no. 4, pp. 447-454, 2016.

[2] I. Dragancea, M. Rundgren, E. Englund, H. Friberg, and T. Cronberg, "The influence of induced hypothermia and delayed prognostication on the mode of death after cardiac arrest," Resuscitation, vol. 84, no. 3, pp. 337-342, 2013.

[3] K. Nagao, H. Nonogi, N. Yonemoto et al., "Duration of prehospital resuscitation efforts after out-of-hospital cardiac arrest," Circulation, vol. 133, no. 14, pp. 1386-1396, 2016.

[4] M. K. Chae, E. Ko, J. H. Lee et al., "Better prognostic value with combined optic nerve sheath diameter and grey-to-white matter ratio on initial brain computed tomography in post-cardiac arrest patients," Resuscitation, vol. 104, pp. 40-45, 2016.

[5] S. P. Choi, H. K. Park, K. N. Park et al., "The density ratio of grey to white matter on computed tomography as an early predictor of vegetative state or death after cardiac arrest," Emergency medicine journal: EMJ, vol. 25, no. 10, pp. 666-669, 2008.

[6] C. Cristia, M. L. Ho, S. Levy et al., "The association between a quantitative computed tomography (CT) measurement of cerebral edema and outcomes in post-cardiac arrest-a validation study," Resuscitation, vol. 85, no. 10, pp. 13481353, 2014.

[7] A. Gentsch, C. Storm, C. Leithner et al., "Outcome prediction in patients after cardiac arrest: a simplified method for determination of gray-white matter ratio in cranial computed tomography," Clinical Neuroradiology, vol. 25, no. 1, pp. 4954, 2015.

[8] U. Hanning, P. B. Sporns, P. Lebiedz et al., "Automated assessment of early hypoxic brain edema in non-enhanced CT predicts outcome in patients after cardiac arrest," Resuscitation, vol. 104, pp. 91-94, 2016.

[9] J. Inamasu, S. Miyatake, M. Nakatsukasa, H. Koh, and T. Yagami, "Loss of gray-white matter discrimination as an early CT sign of brain ischemia/hypoxia in victims of asphyxial cardiac arrest," Emergency Radiology, vol. 18, no. 4, pp. 295298, 2011.

[10] C. H. Jeon, J. S. Park, J. H. Lee et al., "Comparison of brain computed tomography and diffusion-weighted magnetic resonance imaging to predict early neurologic outcome before target temperature management comatose cardiac arrest survivors," Resuscitation, vol. 118, pp. 21-26, 2017.

[11] S. Kim, S. Choi, K. Park, C. Youn, S. Oh, and S. Choi, "Early brain computed tomography findings are associated with outcome in patients treated with therapeutic hypothermia after out-of-hospital cardiac arrest," Scandinavian journal of trauma, resuscitation and emergency medicine, vol. 21, no. 1, p. 57, 2013.

[12] Y. H. Kim, J. H. Lee, C. K. Hong et al., "Feasibility of Optic Nerve Sheath Diameter Measured on Initial Brain Computed Tomography as an Early Neurologic Outcome Predictor After Cardiac Arrest," Academic Emergency Medicine, vol. 21, no. 10, pp. 1121-1128, 2014.

[13] B. K. Lee, K. W. Jeung, H. Y. Lee, Y. H. Jung, and D. H. Lee, "Combining brain computed tomography and serum neuron specific enolase improves the prognostic performance compared to either alone in comatose cardiac arrest survivors treated with therapeutic hypothermia," Resuscitation, vol. 84, no. 10, pp. 1387-1392, 2013. 
[14] B. K. Lee, K. W. Jeung, K. H. Song et al., "Prognostic values of gray matter to white matter ratios on early brain computed tomography in adult comatose patients after out-of-hospital cardiac arrest of cardiac etiology," Resuscitation, vol. 96, pp. 46-52, 2015.

[15] B. K. Lee, W. Y. Kim, J. Shin et al., "Prognostic value of gray matter to white matter ratio in hypoxic and non-hypoxic cardiac arrest with non-cardiac etiology," The American Journal of Emergency Medicine, vol. 34, no. 8, pp. 1583-1588, 2016.

[16] D. H. Lee, B. K. Lee, K. W. Jeung et al., "Relationship between ventricular characteristics on brain computed tomography and 6-month neurologic outcome in cardiac arrest survivors who underwent targeted temperature management," Resuscitation, vol. 129, pp. 37-42, 2018.

[17] K. S. Lee, S. E. Lee, J. Y. Choi et al., "Useful computed tomography score for estimation of early neurologic outcome in post-cardiac arrest patients with therapeutic hypothermia," Circulation journal : official journal of the Japanese Circulation Society, vol. 81, no. 11, pp. 1628-1635, 2017.

[18] Y. H. Lee, Y. T. Oh, H. C. Ahn et al., "The prognostic value of the grey-to-white matter ratio in cardiac arrest patients treated with extracorporeal membrane oxygenation," Resuscitation, vol. 99, pp. 50-55, 2016.

[19] H. Liu, P. Xu, F. He, Y. Liu, and J. Wang, "Neurological prognostic value of gray-white-matter ratio in patients after respiratory and cardiac arrest," Zhonghua wei zhong bing ji jiu yi xue, vol. 29, no. 10, pp. 893-896, 2017.

[20] R. B. Metter, J. C. Rittenberger, F. X. Guyette, and C. W. Callaway, "Association between a quantitative CT scan measure of brain edema and outcome after cardiac arrest," Resuscitation, vol. 82, no. 9, pp. 1180-1185, 2011.

[21] J.-A. Ryu, C. R. Chung, Y. H. Cho et al., "The association of findings on brain computed tomography with neurologic outcomes following extracorporeal cardiopulmonary resuscitation," Critical care, vol. 21, no. 1, p. 15, 2017.

[22] M. Scarpino, F. Lolli, G. Lanzo et al., "Neurophysiology and neuroimaging accurately predict poor neurological outcome within 24 hours after cardiac arrest: the ProNeCA prospective multicentre prognostication study," Resuscitation, vol. 143, pp. 115-123, 2019.

[23] S. H. Son, I. H. Lee, J. S. Park et al., "Does combining biomarkers and brain images provide improved prognostic predictive performance for out-of-hospital cardiac arrest survivors before target temperature management?," Journal of clinical medicine, vol. 9, no. 3, p. 744, 2020.

[24] K. J. Streitberger, C. Endisch, C. J. Ploner et al., "Timing of brain computed tomography and accuracy of outcome prediction after cardiac arrest," Resuscitation, vol. 145, pp. 8-14, 2019.

[25] M. T. Torbey, M. Selim, J. Knorr, C. Bigelow, and L. Recht, "Quantitative analysis of the loss of distinction between gray and white matter in comatose patients after cardiac arrest," Stroke, vol. 31, no. 9, pp. 2163-2167, 2000.

[26] G. N. Wang, X. F. Chen, J. R. Lv, N. N. Sun, X. Q. Xu, and J. S. Zhang, "The prognostic value of gray-white matter ratio on brain computed tomography in adult comatose cardiac arrest survivors," Journal of the Chinese Medical Association: JCMA, vol. 81, no. 7, pp. 599-604, 2018.

[27] C. S. Youn, C. W. Callaway, and J. C. Rittenberger, "Combination of initial neurologic examination, quantitative brain imaging and electroencephalography to predict outcome after cardiac arrest," Resuscitation, vol. 110, pp. 120-125, 2017.
[28] A. Liberati, D. G. Altman, J. Tetzlaff et al., "The PRISMA statement for reporting systematic reviews and meta-analyses of studies that evaluate healthcare interventions: explanation and elaboration," BMJ, vol. 339, no. jul21 1, p. b2700, 2009.

[29] P. F. Whiting, A. W. Rutjes, M. E. Westwood et al., "QUADAS-2: a revised tool for the quality assessment of diagnostic accuracy studies," Annals of Internal Medicine, vol. 155, no. 8, pp. 529-536, 2011.

[30] C. Lopez Soto, L. Dragoi, C. C. Heyn et al., "Imaging for neuroprognostication after cardiac arrest: systematic review and meta-analysis," Neurocritical Care, vol. 32, no. 1, pp. 206216, 2020.

[31] A. Arbelaez, M. Castillo, and S. K. Mukherji, "Diffusionweighted MR imaging of global cerebral anoxia," AJNR. American Journal of Neuroradiology, vol. 20, no. 6, pp. 999-1007, 1999.

[32] Y. Morimoto, O. Kemmotsu, K. Kitami, I. Matsubara, and I. Tedo, "Acute brain swelling after out-of-hospital cardiac arrest: pathogenesis and outcome," Critical Care Medicine, vol. 21, no. 1, pp. 104-110, 1993.

[33] J. Y. Hong, D. H. Lee, J. H. Oh et al., “Grey-white matter ratio measured using early unenhanced brain computed tomography shows no correlation with neurological outcomes in patients undergoing targeted temperature management after cardiac arrest," Resuscitation, vol. 140, pp. 161-169, 2019.

[34] J. H. Oh, S. P. Choi, J. H. Wee, and J. H. Park, "Inter-scanner variability in Hounsfield unit measured by CT of the brain and effect on gray-to-white matter ratio," The American Journal of Emergency Medicine, vol. 37, no. 4, pp. 680-684, 2019. 